\title{
Socioeconomic differences in the utilization of health services in a Dutch population: the contribution of health status
}

\author{
Joost B.W. van der Meer*, Johannes van den Bos, Johan P. Mackenbach \\ Department of Public Health, Erasmus University Rotterdam, P.O. Box 1738, \\ 3000 DR Rotterdam, The Netherlands
}

Received 4 September 1995; revised 12 February 1996; accepted 12 February 1996

\begin{abstract}
The main question addressed here is to what extent socioeconomic differences in the utilization of health services in the Netherlands can be explained by health status. Our aim is to assess whether the health service has achieved equal access for equal needs, and which health status measures best control for need. Cross-sectional survey data from 2867 respondents with respect to utilization of six different types of health service are used for analysis. Socioeconomic differences in utilization were present for all services after we controlled for age, sex and marital status. By controlling for health status, differences changed markedly for all health services analyzed. Differences in general practitioner contacts diminished but did not disappear (adjusted odds ratio primary education/university 2.22). The pattern of excess contacts with specialist physicians reverses (adjusted odds ratio 0.74 ). This is also true for the physiotherapist. The pattern of hospitalizations is unclear. Use of over-the-counter medicines is little affected by control for health status. Adjusted differences in use of prescription medicines become small. Control for health status is best achieved with a set of health measures covering several dimensions of health. Whether low relative utilization among those with low education reflects limited access, or whether higher use of other services is compensatory is hard to decide on the basis of this study. Monitoring access to health care is important for all sorts of systems, including those which are believed to be equitable.
\end{abstract}

Abbreviations: ADL, activities of daily living; CI, confidence interval; COPD, chronic obstructive pulmonary disease; GP, general practitioner; MRC, Medical Research Council; NHP, Nottingham Health Profile; OECD, Organization for Economic Co-operation and Development; OR, Odds ratio; OTC medicines, over-the-counter medicines (as opposed to prescription medicines); PGH, perceived general health.

* Corresponding author. Tel.: + 31104087714 ; fax: + 31104366831 ; e-mail: vandermeer@mgz. fgg.eur.nl.

0168-8510/96/\$15.00 (C) 1996 Elsevier Science Ireland Ltd. All rights reserved

PII S0168-8510(96)00817-2 
Keywords: Social class; Health services utilization; Health services access; Health status measures; Surveys, Netherlands

\section{Introduction}

Every citizen has a right to access to high-quality health care. According to the UN International Covenant on Economic, Social and Cultural Rights participating governments should take steps necessary for 'the creation of conditions which would assure to all medical service and medical attention in the event of sickness' [1].

Access to the health service for all groups in society has been a much-debated issue in the last two decades (and before) in many industrialized countries [2-11]. In the US the debate has generated a considerable body of knowledge through studies on access to health services, for example on socioeconomic differences in health services utilization. These studies show that, under control for health status, the socially disadvantaged have lower physician utilization rates [12,13], lower utilization rates of designated medical procedures [14,15], while length-of-stay in hospitals is longer [16].

Health care and finance in the US, with limited public insurance schemes, is organized very differently compared to most European countries or Canada, where National Health Services or a mixture of public and private insurance exist. Therefore, studies on access to the health care system in the US are of limited generalizability to countries with a different system. Relatively few recent studies have examined the issue of access in countrics outside the US, though there are examples of fairly recent studies about access to the National Health Systems in Great Britain [17,18], Italy [9,19], and the health system of Canada [20,21]. Studies like these usually analyze a limited number of health services. Only one study, a multi-country comparison, covers the complete health system by analyzing total health expenditure [4].

The Dutch Longitudinal Study on SocioEconomic Differences in the Utilization of Health Services (LS-SEDUHS) aims at describing and cxplaining sociocconomic differences in the utilization of a large range of health services in a predominantly chronically ill population in the south-east of the Netherlands. The study could fill some gaps in our knowledge about access to the health care system in a setting with a mixed insurance system. In the Netherlands, people earning less than approximately DFL 58000 (US\$ 35000) have a compulsory public insurance, and those with an income above DFL 58000 are privately insured. Virtually the entire population is covered [22].

The question of interest when studying access is whether the health care system realizes equal access for equal need. When one applies this principle to socioeconomic status, in equal access situations the lower strata (who are less healthy than people in the higher strata $[22,23]$ ) should show higher utilization figures. Theoretically, the gap between socioeconomic groups should disappear when need, i.e. health status, is taken into account. 
When controlling for need, it is of course essential to cover all relevant aspects of health status. Unfortunately, we do not have a systematic insight into the contribution of several dimensions of health status (like perceived health, diseases and disabilities) to the explanation of socioeconomic differences in health service utilization. Theoretically, we would assume that measures covering the above-mentioned three dimensions, rather than just one or two, provide a more adequate representation of medical need. The LS-SEDUHS contains extensive information on health status and thus lends good opportunities to study the contribution of several health status measures.

The main questions addressed in this article are therefore:

What is the contribution of designated health status measures in explaining sociocconomic diffcrences in utilization of health services? Which health status measures are relevant to control for health status when studying socioeconomic differences in health services utilization?

Is there equal utilization for equal need between socioeconomic groups of a broad spectrum of health services (such as contact with a general practitioner, specialist physician, or physiotherapist, hospital admissions, and use of prescription or over-the-counter medicines)?

\section{Data and methods}

\subsection{Study population}

The LS-SEDUHS is part of the GLOBE study, a longitudinal study about incqualitics in health in the Netherlands that started in 1991. The design and objective of this study have been described in detail elsewhere [24]. For the LS-SEDUHS, baseline data from the GLOBE postal survey were used to select the study population. The sample of the GLOBE study is based on a cohort of non-institutionalized Dutch nationals 15-74 years old, oversampling the highest and lowest socioeconomic strata, as well as people aged 45 years and over. For the LS-SEDUHS it was desirable to over-represent people with an illness to obtain sufficient events of health care utilization on a wide range of scrvices. Information on chronic diseases from the GLOBE-questionnaire was used to select all persons reporting chronic obstructive pulmonary disease (COPD) and asthma, cardiac problems, diabetes or severe lower back pain. A random sample of the remainder of the population was drawn to obtain participants without chronic disease, or other than the above four. The four conditions were chosen for three reasons: they constitute a considerable part of the burden of chronic disease, socioeconomic differences in health status can be expected, and validated questionnaires for these conditions exist.

In total, 2867 respondents $(72.2 \%)$ completed a separate mailed questionnaire and subsequent interview. There was no selective response by most socio-demographic characteristics, except for a smaller response rate among people aged $15-34$. Only a slightly smaller response among those in the lowest education classes 
could be detected, and no important differences in response by health status occurred [25]. Some basic data on the composition of the study group are shown in Table 1.

\subsection{Data collection}

The interview and questionnaire contained information concerning a wide array of services: general practitioner contacts and specialist physician contacts during the 2 months preceding the interview, contact with a physiotherapist and hospitalizations in the preceding year, and use of prescription and over-the-counter (OTC) medicines during the past 14 days. Health status measures included disabilities (checklist on constraints of Activities of Daily Living (ADL), short version of the disability indicator of the Organization of European Co-operation and Development (OECD) [26]) and self-rated health (perceived general health (PGH) [27],

Table 1

Composition of study population by age, sex, education and disease status

\begin{tabular}{lcc}
\hline & $n$ & \\
\hline Age & & \\
$14-24$ & 164 & 5.7 \\
$25-34$ & 234 & 8.2 \\
$35-44$ & 305 & 10.6 \\
$45-54$ & 775 & 27.0 \\
$55-64$ & 825 & 28.8 \\
65 and over & 564 & 19.7 \\
Sex & & 51.5 \\
Male & 1476 & 48.5 \\
Femalc & 1391 & 23.9 \\
Education & & 38.4 \\
Primary & 685 & 19.8 \\
Lower vocational, general secondary & 1101 & 11.8 \\
Intermediate vocational, higher secondary & 569 & 3.6 \\
Higher vocational & 338 & 2.4 \\
University & 104 & \\
Other, unknown & 70 & 21.0 \\
Disease status & & 30.2 \\
Asthma, COPD & 603 & 3.1 \\
Heart disease & 867 & 34.7 \\
Diabetes & 231 & 65.5 \\
Low back complaints & 996 & 100.0 \\
Total with at least one of the above four diseases & $1878^{\mathrm{a}}$ & 977 \\
None of the above four & 12 & 2867 \\
Missing data on all four diseases & & \\
Total study population & & \\
\hline & & \\
\hline
\end{tabular}

aCo-morbidity among the four groups was allowed, so this total (representing persons) is less than the total of the four disease groups (representing cases). 
Nottingham Health Profile (NHP) [28]). Respondents also filled out a checklist of chronic conditions.

On the four over-represented conditions, existing validated (Dutch) questionnaires for that condition were used. For asthma/COPD this was a Dutch translation of the British Medical Research Council (MRC) questionnairc [29,30]. The Rose-questionnaire on angina pectoris as well as Dutch material on heart failure were used for heart conditions [31,32]. For diabetes, questions from a Dutch survey were used [33], and a questionnaire for lower back pain was constructed based on the Standardised Nordic questionnaire for this condition and a questionnaire used in a large Dutch health survey $[34,35]$.

Questions concerning health insurance were also included. Socio-demographic variables were marital status (single, marricd, divorced, widowed), sex and age; socioeconomic status was determined by highest attained education (seven classes).

\subsection{Methods}

Of the four over-represented conditions, disease severity was established based on ratings - if present - derived from the original questionnaire. The construction of the stages of scverity of cach of thesc conditions is explained in detail in Appendix A. To show that the developed severity categories are meaningful, their relation with general practitioner (GP) contacts is shown in Table 2. For nearly all categories there is a positive gradient with the utilization of the general practitioner. An analysis with other health services performed likewise (results not shown).

The remainder of the chronic conditions were separately coded as dichotomous variables (absent/present). All other health status measures were coded as polychotomous variables. Marital status was used in the original four categories, age was recorded into twelve 5-year classes. Persons presently following some sort of education were recorded according to their present education instead of allocating them to their highest attained education. The seven categories were collapsed into five.

\subsection{Analyses}

Logistic regression with utilization (yes/no) as dependent variable was done with SAS proc logistic version 6.07 under UNIX [36]. Firstly a basic model was fitted containing socio-demographic confounders. Confounders were selected on their known or suspected association with both socioeconomic status and utilization of health services and, after that, on statistical criteria [37]. Confounders were age, sex, and marital status (model 1). Degree of urbanization and religion were considered as confoundcrs, but appcared to play no significant role. After these socio-demographic confounders, education is added to the model. The reduction in deviance (RD) of education in a regression model was used to test the overall effect of education in that particular model. The RD of education in logistic regression is analogous to the numerator of the partial $F$-test in ordinary least squares regression. 


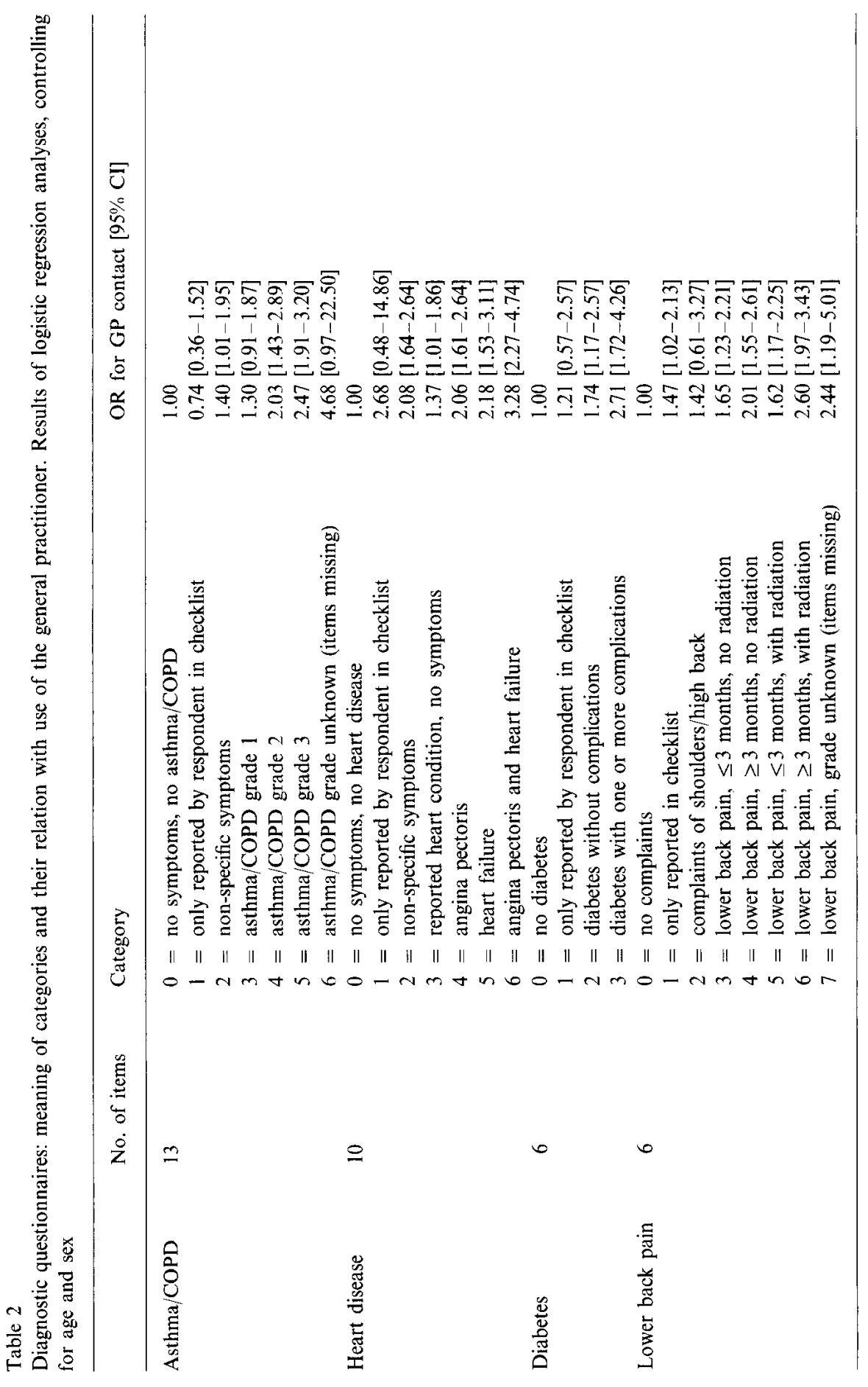


Health status measures were added to model 1 to control for need. 'Objective' health status measures (reported chronic diseases, handicaps and disabilities) were added first (model 2). Also 'subjective' health status measures (health or complaints as perceived by the respondent) were entered into the basic model (model 3). Finally, a model was fitted containing both 'objective' and 'subjective' health status measures (model 4). Education was added to these models. Socioeconomic differences in the utilization of health services are expressed as odds ratios (OR) with the corresponding $95 \%$ confidence intervals for each of the educational classes, taking class 1 (university degree) as reference category $(O R=1)$. If health status variables explain the socioeconomic differential in the utilization of health services, the OR of a particular educational group will shift towards 1 compared to the model without these variables. The shifts were separately tested by a Wald-type collapsibility test statistic proposed by Maldonado and Greenland [37], which tests the shift in the corresponding regression coefficient (beta). Maldonado and Greenland recommend a threshold $P$-value of 0.20 , in order not to miss any important effects.

The effect of each single health status measure or particular set of health status measures in controlling for health was compared with the effect of all measures together. For each situation, the change in beta ('beta-shift') of the lowest educational group was taken as a percentage of the beta shift of a model with all health status measures. The highest educational group is used as reference. These analyses were done separately for all health services considered here.

\section{Results}

Table 3 shows the utilization figures by socioeconomic status for the health services under study. The first column (model 1) shows figures adjusted for sociodemographic confounders only. All services show higher utilization figures by those with lower education compared to those with an academic background, the only exception being OTC drugs which show a reverse pattern. A clear gradient from highest to lowest socioeconomic group is not always present. The only statistically significant odds ratios are those for GP contacts (lower 3 classes), prescription medicines (primary school) and OTC drugs (all groups). For GP contacts, the reduction in deviance (RD) for education is 38.34 with a $P$-value $<$ 0.05 , implying that the overall contribution of education to differences in GP contacts is statistically significant. Similarly, education contributes significantly to differences in hospitalizations, use of prescription drugs and use of OTC drugs.

Control for 'objective' health status measures (model 2) reduces differences for GP contacts, whereas the patterns for seeing a specialist physician or physiotherapist are reversed. Hospitalizations now differ little by socioeconomic status. Also utilization of prescription drugs differs little by socioeconomic status, whereas the pattern of lower use of OTC drugs in lower educational groups is enlarged. Control for 'subjective' health status measures essentially shows the same pattern in a more pronounced way (model 3 ). Control for both categories of health status measures (model 4) more clearly shows the pattern already present in the models 2 or 3 . The 


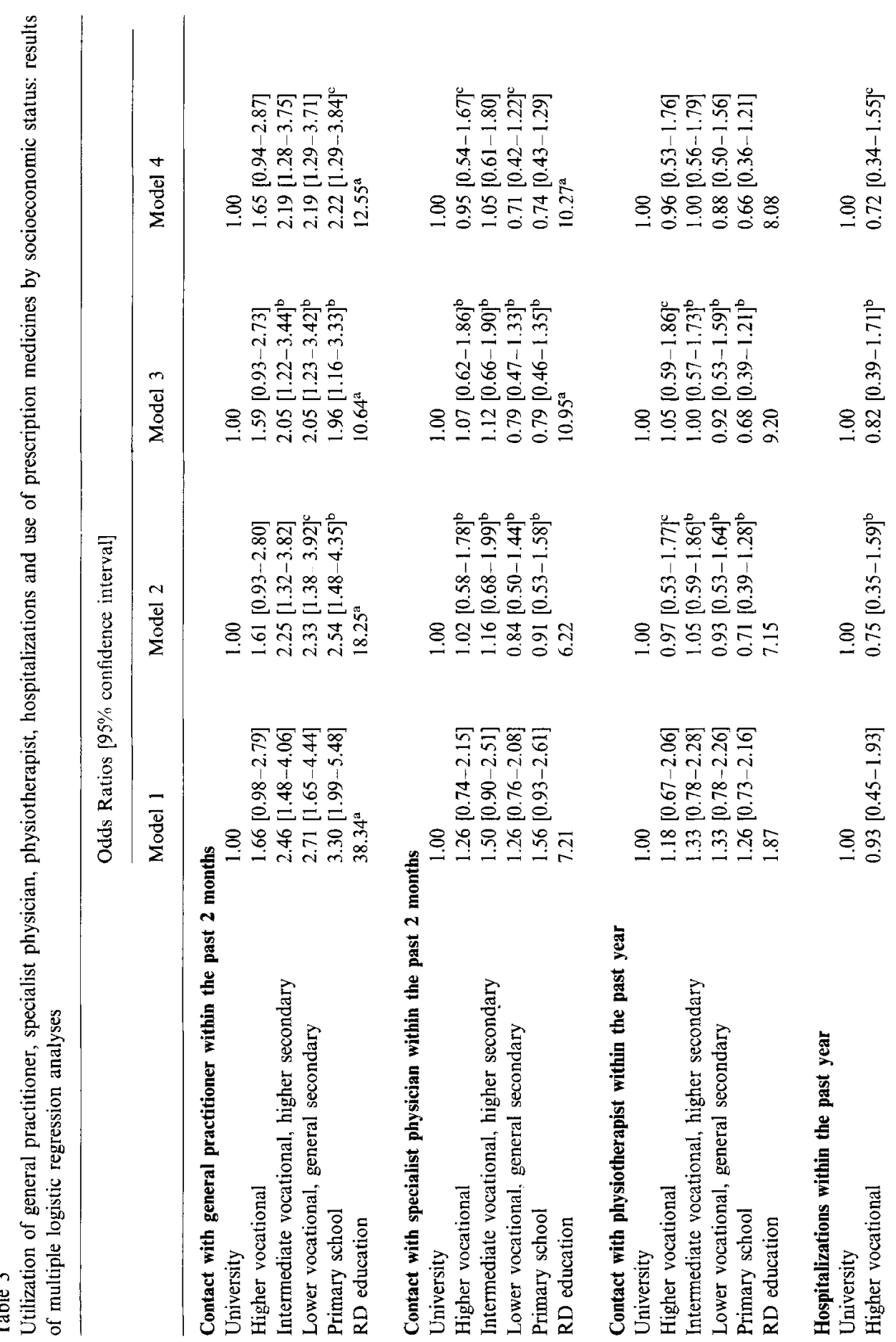




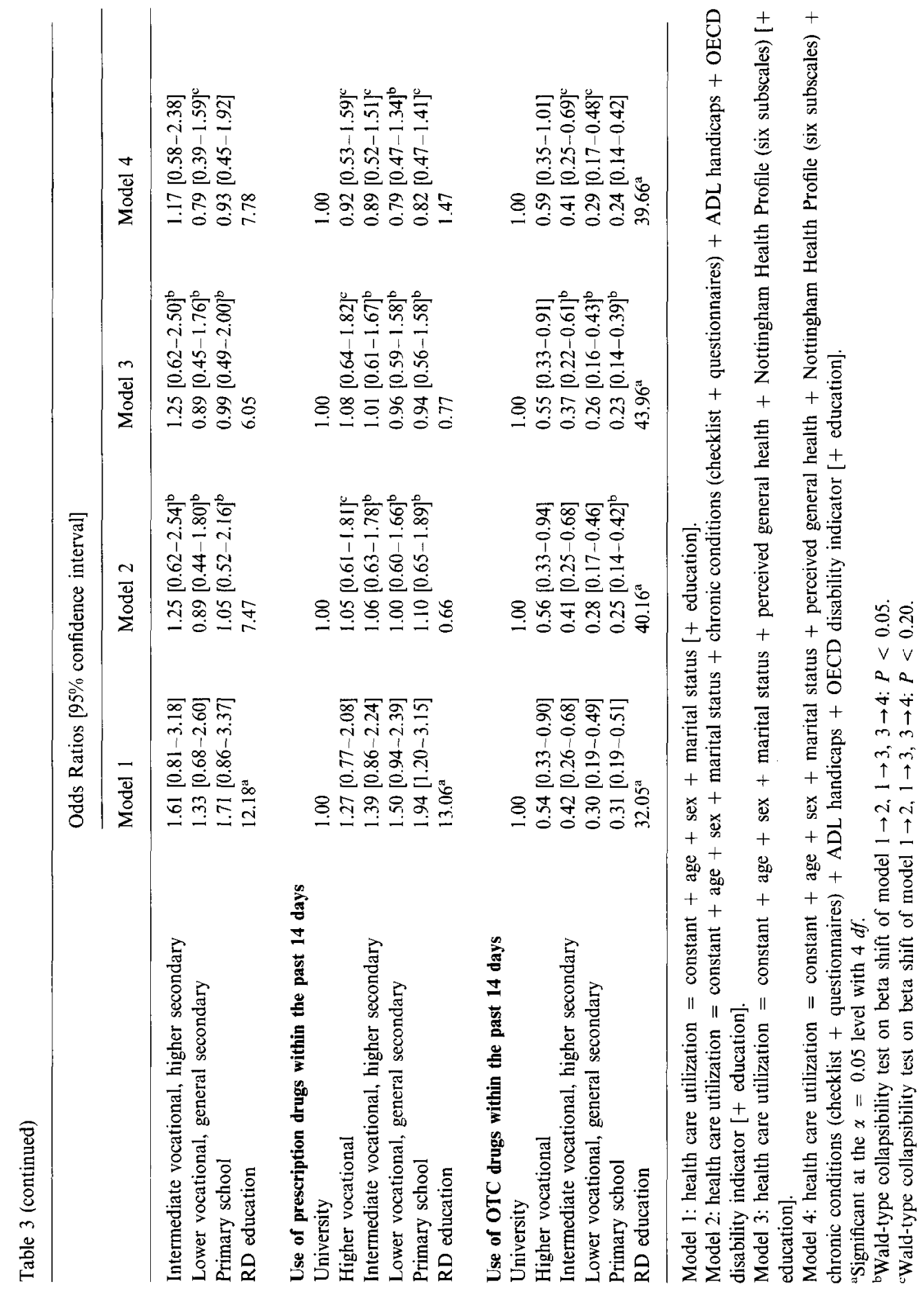


relation between the odds ratios in the four educational groups for each regression model is illustrated graphically for two examples. Fig. 1 shows GP contacts and Fig. 2 shows contact with the specialist physician; models $1-4$ are displayed on the $x$-axis and the $y$-axis displays odds ratios.

Table 4 shows the effect each health status measure has on the beta shift of those with primary school education, as a percentage of the total beta shift in that group if all health status measures were used. PGH in itself is responsible for the largest shift in beta's, except for contacts with the physiotherapist and OTC medicines. By applying just this health status measure, the beta shifts of education range between $58 \%$ and $122 \%$, though most values are mid-range. When using both 'subjective' health status measures, PGH and NHP beta shifts range from $83 \%$ to $131 \%$. For 'objective' health status measures combined the beta shifts range from $65 \%$ to $95 \%$. PGH, chronic diseases and ADL-handicaps were combined to investigate how a set

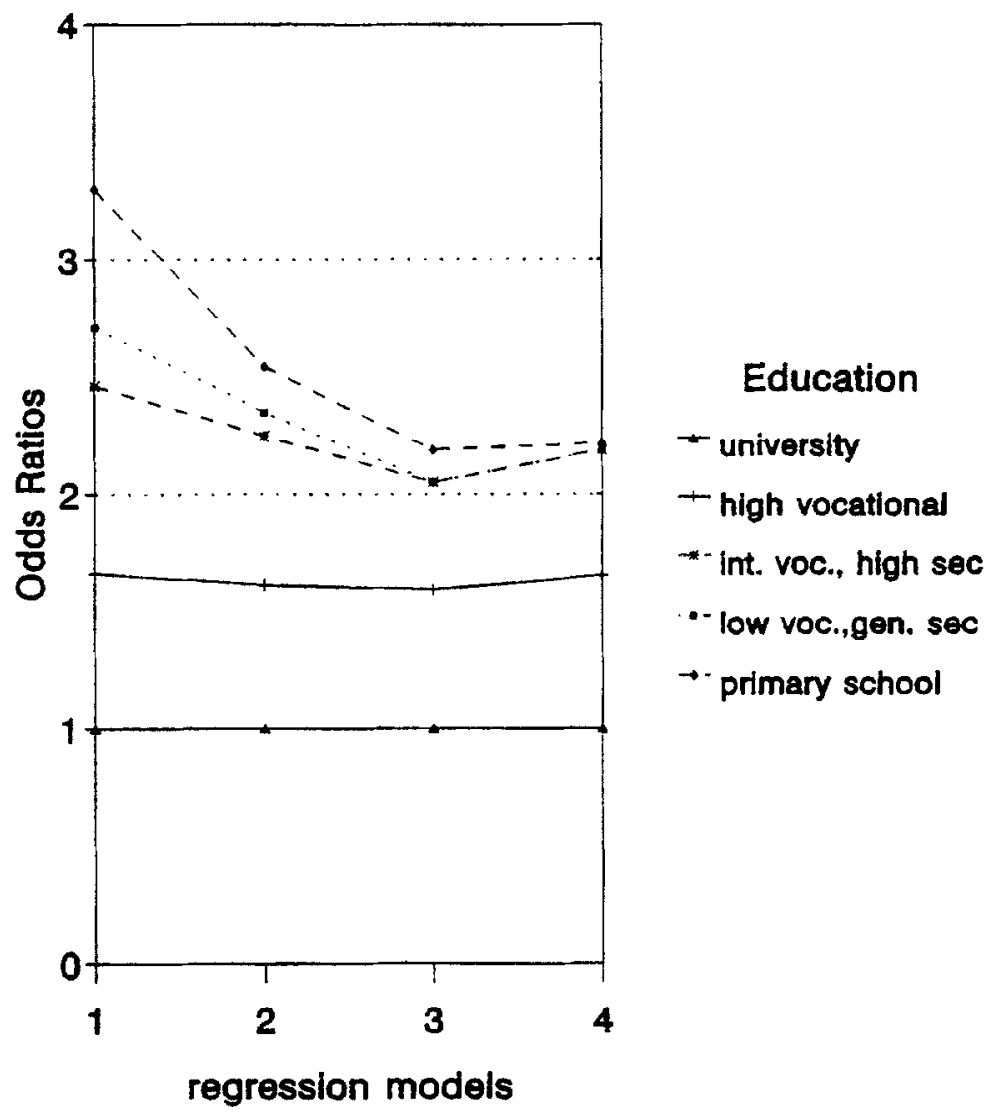

\section{numbers of regression models refer to table 3}

Fig. 1. GP contacts within the past 2 months by education, various regression models. 


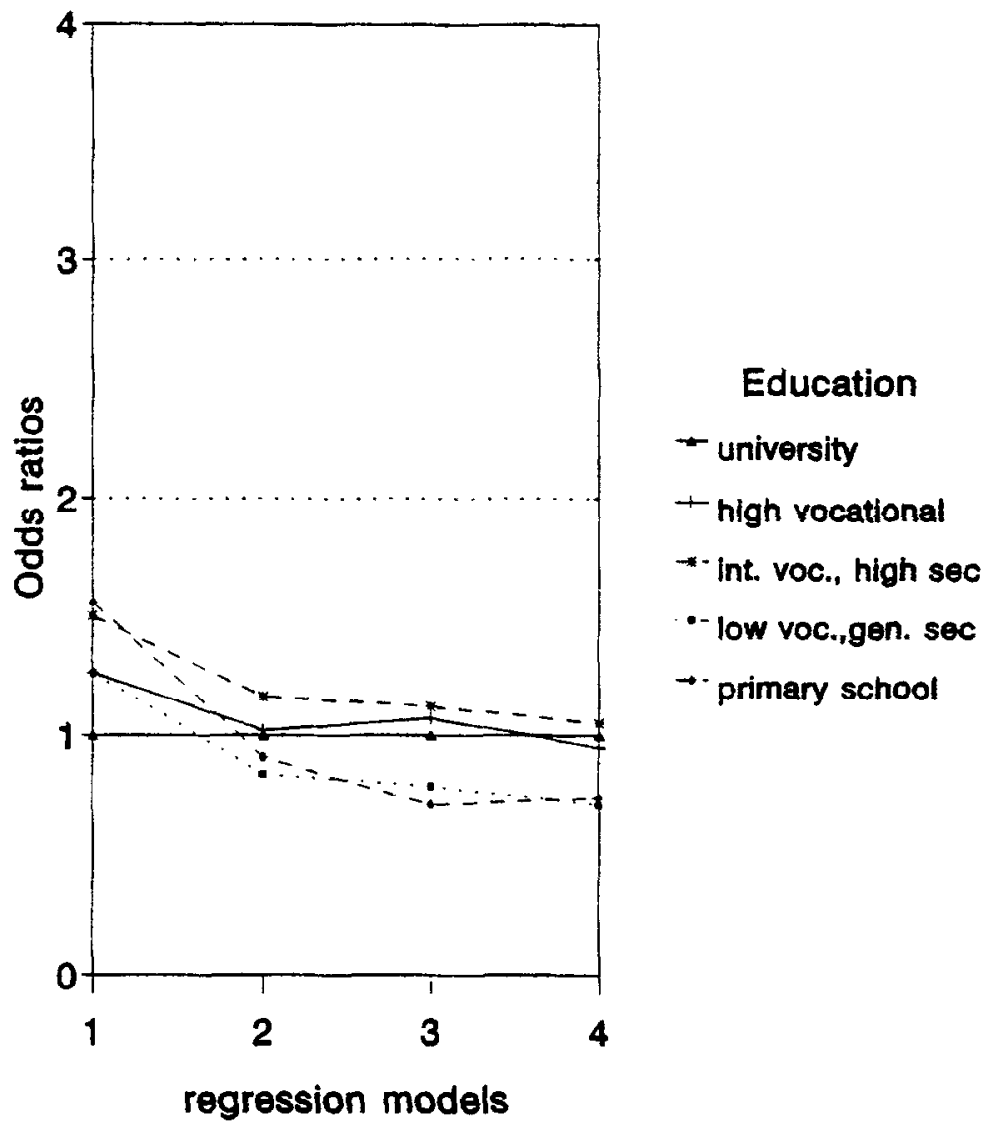

\section{numbers of regression models refer to table 3}

Fig. 2. Specialist contacts within the past 2 months by education, various regression models.

of health status measures would perform, each covering one separate dimension of health status (subjective health, disease, disabilities). The beta shift for this combination ranges from $88 \%$ to $103 \%$.

\section{Discussion}

In a cross-sectional analysis of Dutch survey data of 2867 persons, we have demonstrated socioeconomic differences in the utilization of health services after we controlled for age, sex and marital status: all services - except the use of over-the-counter (OTC) drugs — are used more frequently by the less educated. 


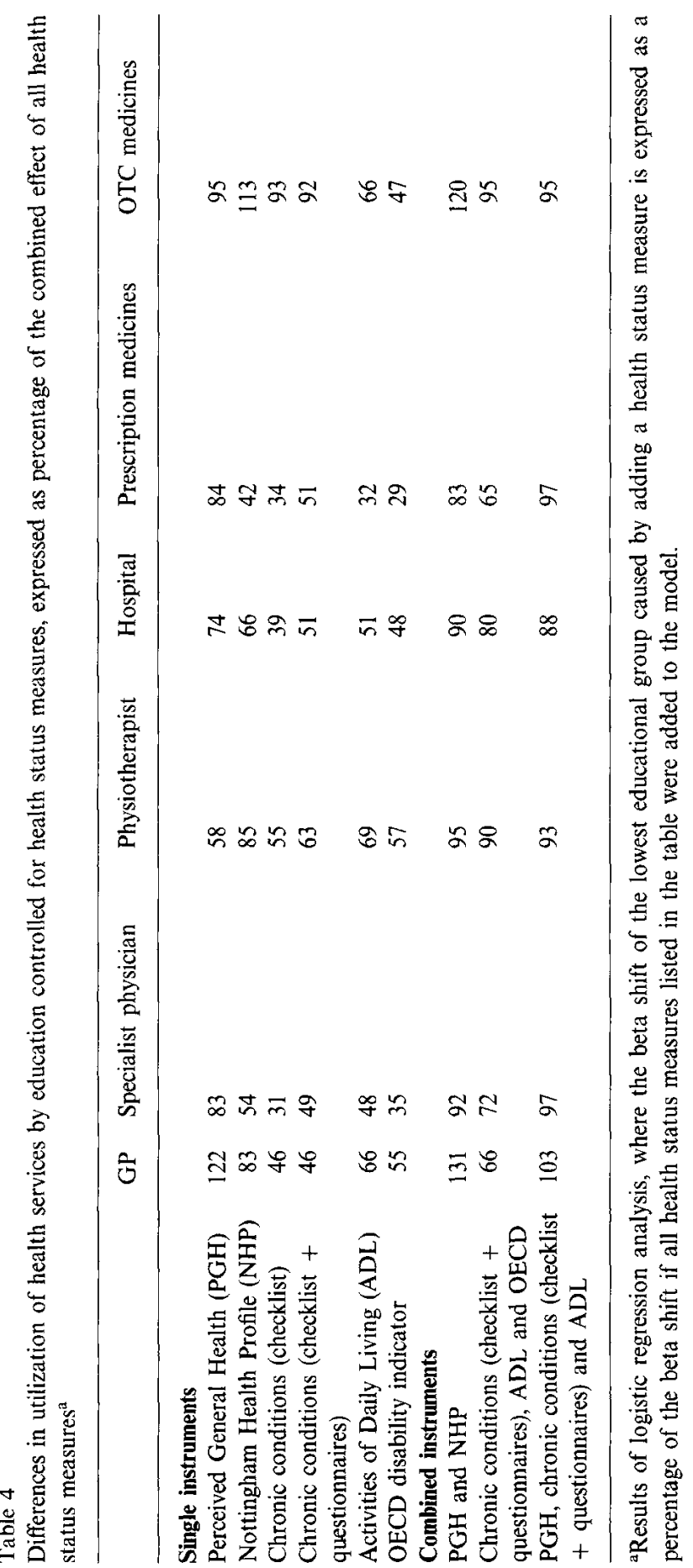


After controlling for health status, these differences changed markedly for all health services analyzed. Some differences were reduced (GP contacts), other differences reversed (e.g. specialist physician). The odds ratios of the lowest educational class for GP contacts, for example, changed from 3.30 [95\% CI: $1.99-5.48]$ to 2.22 [95\% CI: $1.29-3.84]$. The odds ratios of the lowest educational class for contacts with a specialist physician changed from $1.56[95 \% \mathrm{CI}: 0.93-2.61]$ to 0.74 [95\% $\mathrm{CI}$ : $0.43-1.29]$. Five different health status measures were used, and their impact on the size of socioeconomic differences in health care utilization was calculated. The impact of a single health measure depends on the type of health service considered, but is usually $40-70 \%$ of the impact of the five measures together. Perceived General Health (PGH) had the largest impact, and the 'subjective' health status measures PGH and Nottingham Health Profile (NHP) together had a larger impact than all 'objective' health status measures together (chronic conditions, disabilities and handicaps).

When interpreting the data some limitations of the study design have to be considered. The study is entirely based on survey data, i.e. on information provided by the respondent about chronic conditions and health care utilization. Results might be different when data from other databases, e.g. hospital records are used. Bias will only occur if groups with different socioeconomic status also report differentially about their utilization of health services or health status. There is some evidence that lower socioeconomic groups systematically underreport certain conditions, such as cancer [38], COPD/asthma and heart disease [39]. This would imply an underestimation of the socioeconomic differences in health, and hence insufficient control for health status. However, for the majority of the respondents we were able to reach a diagnosis by specific questionnaires, rather than by a checklist of chronic conditions.

The occurrence of selection bias where ill people with a low socioeconomic status and not having access to the health care system are under-represented in our study is a possibility. However, in these data there are no major differences in response by socioeconomic status and illness level [25]. The percentage of uninsured in the study population is smaller than in the Dutch population as a whole, but as both proportions are very small [40], this will hardly cause any bias.

It should be noted that the majority of persons have been selected for their reporting of one or more chronic conditions. Results therefore cannot be generalized to a healthy population, although the observed patterns are similar to those from the Netherlands Health Interview Survey, which is representative for the Dutch population [27].

The results indicate that the use of one health status measure to control for health status may be insufficient when socioeconomic differences in utilization of health services are analyzed (Table 4). The best single measure is PGH, which does rather well with most aspects of health service utilization. Sometimes PGH accounts for more than $100 \%$ of the impact of all measures together. The reason for this high percentage is perhaps 'overcontrolling' for health, because the lower socioeconomic groups could be more inclined to judge the same health status as 'bad' as their counterparts with a high socioeconomic status, or are more inclined 
to complain about their health. This mechanism is corrected when other, more 'objectively' measured dimensions like handicaps are taken into account.

The results of the 'subjective' measures PGH plus NHP together do not differ much from those with the full model including 'objective' health status measures. This is probably due to the nature of the NHP, which is a real 'profile' with six subscales. The six subscales not only measure subjective complaints and well-being, but also mobility which will parallel the OECD and ADL-scales on disabilities. This idea is supported by figures of the model covering perceived health, diseases and disability together, measured by different instruments. Chronic diseases, ADL-handicaps and PGH put together show figures very similar to a model with PGH and NHP (Table 4).

$\Lambda$ combination of health status measures covering the three important dimensions of health - perceived complaints, diseases, and handicaps - enables extensive control for health status in surveys aiming at measuring socioeconomic differences in health care utilization.

The results show that socioeconomic differentials in the use of health services are present in this Dutch study population under control for health status. The findings do not necessarily imply that the health care system in the Netherlands is inequitable. In fact, previous research suggests that the health care system in the Netherlands is rather equitable compared to other European countries [4].

The fewer specialist contacts in the low education groups while the reverse is true for contact with the general practitioner is perhaps partly to be explained by a substitution phenomenon in the Dutch system. Going to a specialist physician is financially more attractive for those with private insurance (and consequently a high socioeconomic status), because a substantial part of them have no coverage for the general practitioner. The publicly insured are completely covered for GP services. Although the general practitioner is the gate-keeper in the Dutch health care system, the private sector adheres less strictly to this rule than the public sector.

However, the difference between utilization of general practitioner and specialist may also have other reasons. Attitude differences between social groups in seeking medical attention may be one of them. Independent of health status, people with a low socioeconomic status may be inclined to see a GP relatively often with minor complaints that do not warrant referral. The high figures on OTC medicines in the highly educated groups while socioeconomic differences in taking prescription drugs are small, could be another aspect of a difference in attitude: those with higher education might be more inclined to try to alleviate minor complaints without seeking professional help. Referral is not only determined by the severity of the complaints. Some (higher educated) patients who believe themselves to be better off with a specialist could press the GP to refer, while the complaint can be perfectly dealt with by the GP.

Instead of these general explanations, differences in access to the specialist physician may have explanations on a more specific level. Socioeconomic differences have been described with regard to several cardiac procedures, not only in the United States but also in the United Kingdom [17,18,41]. Such differences could also occur in the Netherlands and should be the object of further study. 
Some of the previous alternative explanations are examples of substitution resulting in equivalent care, while other explanations (e.g. pressure to refer to a specialist by those with high education) in fact imply some form of unequal access. To enable reliable inferences about equal access, excellent control for differences in need is indispensable, because without sufficient control for health status observed differences may be attributed to other factors while in fact they are explained by differences in need. It seems wise to use a range of health status measures in surveys to achieve good control for health status, because in different sectors of the health care system different dimensions of health status are important.

Study of determinants of utilization of both general and specific health services across social groups will give policy makers more understanding about how to maintain and improve equal access to health services for all groups in society. This is vital in this era of health care reform, for countries with state-controlled health care systems as well as for countries where the health care system is market-driven.

\section{Acknowledgements}

This project was supported by a grant from the Health Insurance Executive Board (Ziekenfondsraad). It is part of the GLOBE-study (Gezondheid en LevensOmstandigheden Bevolking Eindhoven en omstreken), a large-scale research project on health and living conditions of the population of Eindhoven and surroundings. The GLOBE-study is conducted by the Department of Public Health, Erasmus University Rotterdam, in collaboration with the Community Health Services of the city of Eindhoven, the region of Geldrop-Valkenswaard, and the region of Helmond. The GLOBE-study is supported financially by the Ministry of Welfare, Health and Cultural Affairs, the Health Insurance Executive Board, the Netherlands Organization for Scientific Research (NWO), the National Committee on Chronic Diseases (NCCZ) and the Erasmus University Rotterdam.

\section{Appendix A: Criteria for the severity of the four over-represented conditions}

\section{A.1. Asthma/COPD}

The number of items used to establish severity in the Asthma/COPD questionnaire was 13. Criteria were: period of coughing lasting at least 3 months a year, and/or period of productive cough lasting at least 3 weeks a year, and/or attacks of shortness-of-breath and/or wheezing, and/or shortness-of-breath in rest and on exertion. Staging was based on the number of these symptoms and their combination with shortness-of-breath, resulting in three grades for asthma/COPD.

\section{A.2. Heart conditions}

The number of items used to establish angina pectoris was 10. Criteria were: a heavy feeling in the chest, and/or chest pain or discomfort, and/or attack of pain in 
the jaw, throat, fingers or shoulders on exertion, disappearing in rest or reacting on medication. Heart failure was established by a combination of at least two positive responses to questions on swollen legs, nocturia, and orthopnoea, or shortness-ofbreath (in the absence of asthma/COPD).

\section{A.3. Diabetes}

For diabetes the diagnosis and staging was based on six items. The difference between type I and type II diabetes was ignored. Severity was established using the respondent's positive response to the symptoms of one or more diabetic complications: pain in the legs/badly healing leg ulcers (peripheral vascular complications), numb feeling when walking, difficulty in fastening buttons (polyneuropathy). This resulted in two stages: diabetes without complications, and diabetes with one or more complications.

\section{A.4. Lower back complaints}

The staging for back complaints was based on six items, using prognostic criteria derived from the medical literature, such as radiation of pain to the legs and duration of symptoms longer than 3 months [42], resulting in four stages.

Respondents reporting one of the specified condition(s) who did not meet any of the criteria were given a separate code, as it is likely that their health status is different from someone reporting no condition at all. Also respondents who did not report suffering from one of the mentioned diseases, but who reported symptoms not severe enough to meet any of the diagnostic criteria, were given a separate code for the same reason. The resulting coding for the four over-represented disease categories is listed in Table 2.

\section{References}

[1] The International Bill of Human Rights, International Covenant on Economic, Social and Cultural Rights, United Nations, New York, 1985.

[2] Graham, S., Socio-economic status, illness, and the use of medical services, Milbank Memorial Fund Quarterly, 35 (1957) 58-66.

[3] Townsend, P., Inequality and the health service, Lancet, 868 (1974) 1179-1189.

[4] van Doorslaer, E.A., Wagstaff, A. and Rutten, F.F.H., Equity in the Finance and Delivery of Health Care, Oxford Medical Publications, Oxford, 1993.

[5] Rice, D.P., Ethics and equity in US health care: the data, International Journal of Health Services, 21 (1991) 637-651.

[6] Rein, M., Social class and the health service, New Society, 14 (1969) 803-811.

[7] Aday, L.A. and Andersen, R.M., The national profile of access to medical care: where do we stand? American Journal of Public Health, 74 (1984) 1331-1339.

[8] Serange-Fonterme, R., Social inequalities in medical consumption (in French), Social Science and Medicine, 21 (1985) 103-113.

[9] Piperno, A. and Di Orio, F., Social differences in health and utilization of health services in Italy, Social Science and Medicine, 31 (1990) 305-312. 
[10] Collins, E.M. and Klein, R., Equity and the NHS: self-reported morbidity, access, and primary care, British Medical Journal, 281 (1980) 1111-1115.

[11] Blaxter, M., Equity and consultation rates in general practice, British Medical Journal, 288 (1984) 1963-1967.

[12] Kleinman, J.C., Gold, M. and Makuc, D., Use of ambulatory care by the poor: another look at equity, Medical Care, 19 (1981) $1011-1029$.

[13] Mutchler, J.E. and Burr, J.A., Racial differences in health and health care service utilization in later life: the effect of socioeconomic status, Journal of Health and Social Behavior, 32 (1991) $342-356$.

[14] Gaylin, D.S., Held, P.J., Port, F.K., Hunsicker, L.G., Wolfe, R.A., Kahan, B.D., Jones, C.A. and Agodoa, L.Y.C., The impact of comorbid and sociodemographic factors on access to renal transplantation, Journal of the American Medical Association, 269 (1993) 603-608.

[15] Wenneker, M.B. and Epstein, A.M., Racial inequalities in the use of procedures for patients with ischemic heart disease in Massachusetts, Journal of the American Medical Association, 261 (1989) 253-257.

[16] Epstein, A.M., Stern, J.S. and Weissman, R.S., Do the poor cost more? A multihospital study of patients' socioeconomic status and use of hospital resources, New England Journal of Medicine, 322 (1990) 1122-1128.

[17] Kee, F., Gaffney, B., Currie, S. and O'Reilly, D., Access to coronary catheterisation: fair shares for all? British Medical Journal, 307 (1993) 1305-1307.

[18] Ben-Shlomo, Y. and Chaturvedi, N., Assessing equity in access to health care provision in the UK: does where you live affect your chances of getting a coronary artery bypass graft?, Journal of Epidemiology and Community Health, 49 (1995) 200-204.

[19] Mapelli, V., Health needs, demand for health services and expenditure across social groups in Italy: an empirical investigation, Social Science and Medicine, 36 (1993) 999-1009.

[20] Manga, P., Broyles, R.W. and Angus, D.E., The determinants of hospital utilization under a universal public insurance program in Canada, Medical Care, 25 (1987) 658-670.

[21] Newbold, K.B., Eyles, J. and Birch, S., Equily in health care: methodological contributions to the analysis of hospital utilization within Canada, Social Science and Medicine, 40 (1995) 1181-1192.

[22] Mackenbach, J.P., Socioeconomic health differentials in the Netherlands: a review of recent empirical findings, Social Science and Medicine, 34 (1992) 213-226.

[23] Townsend, P., Davidson, N. and Whitehead, M. (Eds.), Inequalities in Health (The Black Report and The Health Divide), Penguin Books, London, 1988.

[24] Mackenbach, J.P., van de Mheen, H. and Stronks, K., A prospective cohort study investigating the explanation of socio-economic inequalities in health in the Netherlands, Social Science and Medicine, 38 (1994) 299-308.

[25] van der Meer, J.B.W., Looman, C.W.N. and Mackenbach, J.P., The Longitudinal Study on Socioeconomic Differences in Utilization of Health Services (in Dutch), Department of Public Health, Erasmus University Rolterdam, Rotterdam, 1993.

[26] McWhinnie, J.R., Disability assessment in population surveys: results of the OECD common development effort, Revue d' Epidemiologie et Santé, 29 (1981) 421-429.

[27] Central Bureau of Statistics, Netherlands Health Interview Survey 1981-1991, SDU Publishers/ CBS Publications, The Hague, 1992.

[28] Hunt, S.M., McEwen, J. and McKenna, S.P., Measuring Health Status, Croom Helm, London, 1986.

[29] van der Lende, R., Jansen-Koster, E.J., Knijpstra, S., Meinesz, A.F., Wever, A.M.J. and Orie, N.G.M., Definition of asthma/COPD in epidemiology and prevention (in Dutch), Nederlands Tijdschrift voor Geneeskunde, 119 (1975) 1975-1987.

[30] du Florey, C. and Leeder, S.R., Methods for Cohort Studies of Chronic Airflow Limitation, WHO Regional Publications, European Series no. 12, WHO, Copenhagen, 1982.

[31] Rose, G. and Blackburn, H., Cardiovascular Survey Methods, WHO Monograph, 56 (1968) 1-88.

[32] Baart, A., The Older Dock-worker, Aspects of His Cardiorespiratory Condition [thesis] (in Dutch), Erasmus University, Rotterdam, 1973. 
[33] Pennings van der Eerden L., Self-care and motivation of patients with diabetes mellitus (in Dutch), Vakgroep Algemene Gezondheidszorg en Epidemiologie: University of Utrecht, Utrecht, 1984.

[34] Kuorinka, I., Jonsson, B., Kilbom, A., Vinterberg, H., Biering-Sörensen, F., Anderson, G. and Jörgensen, K., Standardised Nordic Questionnaires for the analysis of musculoskeletal symptoms, Applied Ergonomics, 18.3 (1987) 233-237.

[35] Department of Epidemiology and Biostatistics, Questionnaire ERGO-survey (in Dutch), Erasmus University Rotterdam, Rotterdam, 1989.

[36] SAS Institute Inc., SAS/STAT User's Guide, Version 6, 4th edn., Vol. 2, SAS Institute Inc, Cary, NC, 1989.

[37] Maldonado, G. and Greenland, S., Simulation study of confounder-selection strategies, American Journal of Epidemiology, 138 (1993) 923-935.

[38] Schrijvers, C.T.M., Stronks, K., van de Mheen, D.H., Coebergh, J.W.W. and Mackenbach, J.P., Validation of cancer prevalence data from a postal survey by comparison with cancer registry records, American Journal of Epidemiology, 139 (1994) 408-414.

[39] Mackenbach, J.P., Looman, C.W.N. and van der Meer, J.B.W., The effect of differential misreporting of chronic conditions on inequalities in morbidity by level of education. American Journal of Public Health (in press).

[40] Anonymous, The uninsured against health-care expense 1985-1991: an update (in Dutch), Maandbericht Gezondheid, 9 (1992) 18-19.

[41] Findlay, I.N., Dargie, H.J. and Dyke, T., Coronary angiography in Glasgow: relation to coronary heart disease and social class, British Heart Journal, 66 (1991) 70.

[42] Frymoyer, J.W., Back pain and sciatica, New England Journal of Medicine, 318 (1988) 291-300. 